\title{
Myeloid sarcoma associated with blast crisis in chronic myelogenous leukemia - Case Report
}

\author{
Agnieszka Szymczyk ${ }^{1,2}$, Monika Podhorecka', Waldemar Tomczak ${ }^{1,3}$, Justyna Szumiło ${ }^{4}$, \\ Marek Hus ${ }^{1}$ \\ ${ }^{1}$ Chair and Department of Haematooncology and Bone Marrow Transplantation, Medical University, Lublin, Poland \\ 2 Independent Clinical Transplantology Unit, Medical University, Lublin, Poland \\ ${ }^{3}$ Chair of Internal Medicine and Department of Internal Medicine in Nursing, Medical University, Lublin, Poland \\ ${ }^{4}$ Department of Pathomorphology, Medical University, Lublin, Poland
}

Szymczyk A, Podhorecka M, Tomczak W, Szumiło J, Hus M. Myeloid sarcoma associated with blast crisis in chronic myelogenous leukemia - Case Report. J Pre-Clin Clin Res. 2016; 10(2): 136-139. doi: 10.5604/18982395.1227573

\begin{abstract}
I Abstract
Introduction. Myeloid sarcoma (MS) is a rare extramedullary tumour which may precede or occur concomitantly with bone marrow involvement in acute myeloid leukemia, myelodysplastic syndrome, or blast crisis in chronic myeloproliferative disorder. Myeloid sarcoma is most commonly found in lymph nodes, skin, subcutaneous tissue and gums, while it is less common in bones, the retroperitoneal space and eye socket.

Case Report. The case is reported of a 65-year-old woman with chronic myelogenous leukemia treated for about 20 years with hydroxyurea, 6-mercaptopurine and tyrosine-kinase inhibitors. During the treatment, the general condition of the patient progressively deteriorated, lymphadenopathy and splenomegaly worsened, and blast crisis was diagnosed. After the first cycle of induction chemotherapy, the patient's lymph nodes were swollen and painful. One of the lymph nodes was subjected to histopathology, on the basis of which a diagnosis of MS was made. As the patient showed no response to the treatment, palliative care was initiated. Three months after the diagnosis of MS, the disease progressed. The patient died of infectious complications.

Conclusions: A diagnosis of MS, which is considered an adverse prognostic factor, significantly reduces the chances of remission and overall survival in patients with acute myelogenous leukemia or blast crisis inchronic myeloproliferative disorders. It seems that early confirmation of the diagnosis and initiation of the treatment adjusted to the patient's clinical condition may improve the prognosis and increase the response rates.
\end{abstract}

\section{Key words}

myeloid sarcoma, chronic myelogenous leukemia, blast crisis

\section{INTRODUCTION}

Myeloid sarcoma (MS) was first described in 1811 by Burns [1] and involved a lacrimal gland. Since that time, attempts to broaden knowledge about the biology and course of the disease have been made. In 1853, King [2] gave the tumour the name chloroma (due to its green colour, attributed to the peroxidase). The link between MS and acute myelogenous leukemia was first recognized by Dock and Warthin in 1893 [3]. Due to the fact that approximately $30 \%$ of these tumours are white, grey, or brown, in 1967 Rappaport [4] introduced the term granulocytic sarcoma. The name was commonly used until 2002, but since not all tumours are composed of granulocytary cells, the term myeloid sarcoma is favoured today [5].

MS is considered a rare disease with an estimated incidence of 2 per million in adults and 0.7 per million in children. There are only isolated cases or small groups of patients with MS reported in the literature which mostly comprises retrospective studies [5].

The tumor occurs in all age groups. It is most often diagnosed in patients with the recurrence of acute myelogenous leukemia (AML), but it can also precede the development

\footnotetext{
Address for correspondence: Agnieszka Szymczyk, MD, MSc, Department of Haematooncology and Bone Marrow Transplantation, Medical University, Staszica 11, 20-081 Lublin, Poland

e-mail: agnieszka.szymczyk.med@wp.pl

Received: 29 February 2016; accepted: 21 November 2016
}

of the disease. There are also reports of myeloid sarcoma in the course of transformation of myelodysplastic syndromes, blast crisis in chronic myelogenous leukemia, myelofibrosis and polycythemia vera. The disease may manifest as a single or multiple lesions (about $50 \%$ of all cases) $[4,6]$.

\section{OBJECTIVE}

The objective of this study is to present the case of a patient with MS associated with blast crisis in chronic myelogenous leukemia. The patient was diagnosed and treated in the Department of Haematooncology and Bone Marrow Transplantation in Lublin. Description of this rare and difficult to diagnose extramedullar tumour, as well as a literature review, may be of great help in clinical practice.

\section{CASE REPORT}

A woman, born in 1949, was referred from the Gynaecology Ward to the Haematology Clinic in January 1986 due to extreme leukocytosis. A complete blood cell count revealed a WBC of $118 \times 10^{3} / \mathrm{ll}$, RBC $4740 \times 10^{6} / \mathrm{ul}$, PLT $394 \times 10^{3} / \mathrm{ul}$ and $\mathrm{Hgb}$ of $14 \mathrm{~g} / \mathrm{dl}$. A bone marrow aspirate smear showed polychromatic normoblasts (1\%), myeloblasts (1\%), promyelocytes (4\%), neutrophilic myelocytes $(11 \%)$, neutrophilic metamyelocytes (19\%), banded neutrophils (22\%), 
segmented neutrophils (38\%), basophils (1\%), lymphocytes (3\%) and decreased levels of leukocyte alkaline phosphatase (LAP score of 8). Abdominal ultrasound examination showed splenomegaly (spleen size of $140 \times 140 \times 200 \mathrm{~mm}$ ), whereas physical examination did not reveal enlarged lymph nodes. Sokal score was 0.78 . Hydroxycarbamide (1,500 mg/day), and allopurinol (as prophylaxis of tumour lysis syndrome) were administered. Since the patient did not adhere to the doctor's advice, the dosage of hydroxycarbamide was temporarily raised (up to $3,500 \mathrm{mg} /$ day). This resulted in improved parameters of the white blood cell system. Due to the young age of the patient, allogeneic haematopoietic stem cell transplantation was considered, but the patient did not consent to the treatment. For this reason, the existing therapy was continued and 6-mercaptopurine was sometimes used at the dose of 50-100 mg/day.

At the end of 2004, anaemia and leukocytosis became aggravated in spite of an increased dosage of hydroxycarbamide. Progressive splenomegaly was found in the physical examination. The general condition of the patient deteriorated gradually. She complained of asthenia, night sweats and temporary abdominal pain. The therapy with imatinib, at the dose of $400 \mathrm{mg} / \mathrm{day}$, was initiated in September 2007. Results of the treatment included improved parameters of the white blood cell system and reduction in spleen size. Despite treatment, cytogenetic remission was not achieved.

In January 2009, when BCR-ABL transcript levels were $50.5 \%$, the dosage of imatinib was increased to $600 \mathrm{mg} /$ day. In April 2009, when BCR-ABL transcript levels increased to 78.04\% and G250E and M351T mutations were identified, treatment with dasatinib, a second-generation tyrosine kinase inhibitor, was started at the dose of $100 \mathrm{mg} /$ day.As a result, BCR-ABL transcript levels decreased to $16.84 \%$. However, in December 2011, T315I mutation was detected and the treatment with tyrosine kinase inhibitor had to be discontinued. The therapy with hydroxycarbamide and interferon- a was restarted. Due to intense bone pain, interferon- $\alpha$ was discontinued in March 2012 and hydroxycarbamide was used in monotherapy.

During the treatment, the general condition of the patient deteriorated gradually. She felt generally unwell, complained of general bone pain and exercise intolerance. In October 2014, the complete blood count showed an increase in leukocyte count to $89.59 \times 10^{3} / \mathrm{ul}$, whereas physical examination revealed lymphadenopathy of the right supraclavicular nodes (matted into a mass the size of $2 \times 4 \mathrm{~cm}$, painless to palpation). The treatment with 6-mercaptopurine, at the dose of $100 \mathrm{mg} /$ day, was started. Due to the suspicion of blast crisis, the patient was referred to the Haematooncology Department for a one-day inpatient hospital stay in order to perform a bone marrow biopsy. Immunophenotyping of the bone marrow showed that $21 \%$ of the cells were blasts expressing CD33+, CD117+, CD34+, DR+, CD13+- (12\%), MPO+- (12\%), CD65-, CD14-. For this reason, a chemotherapy regimen consisting of $2 \mathrm{CdA}+\mathrm{LD}-\mathrm{Ara}-\mathrm{C}$ (cladribine $8 \mathrm{mg} /$ day on days $1-5$, cytarabine $40 \mathrm{mg}$ /day on days 1-7) was used. After the cytostatic treatment, the peripheral lymph nodes (supraclavicular and axillary) became enlarged and painful. Paracetamol and phenylbutazone ointment were administered, but, due to increasing pain, the patient received Tramadol at the dose of 200-400 mg/day, and Oxycodone Hydrochloride at the dose of 20-40 mg/day. One of the

lymph nodes was biopsied and sent to pathology. However, difficulties encountered in sampling made it impossible to establish a diagnosis.

In January 2015, the intensive cytostatic treatment was discontinued because the chemotherapy with $2 \mathrm{CdA}+\mathrm{LD}-$ Ara-C was ineffective (WBC $191.6 \times 10^{3} / \mathrm{ul}$ ). The patient was given palliative chemotherapy with hydroxycarbamide at the dose of 3,000 mg/day and 6-mercaptopurine at the dose of $100 \mathrm{mg} /$ day.

During her hospital stay, the patient suffered from painful lymphadenopathy. For this reason, a lymph node biopsy was scheduled. Histopathological examination of the sample (Fig. 1) showed: diffuse infiltration of large cells with abundant eosinophilic cytoplasm and round nuclei with single large nucleoli, numerous mitotic figures and apoptotic bodies. Infiltration of the lymph node capsule and perinodular fat were also seen. Immunohistochemistry of the tumour cells showed that they were CD34+, MPO+ (some of them), CD45 (+), CD10(-), and CD117 (-). Ki67 proliferation index (MIB-1) was about 60\%. The diagnosis of MS was made on the basis of clinical picture and histopathological examination of the lymph node.

HES

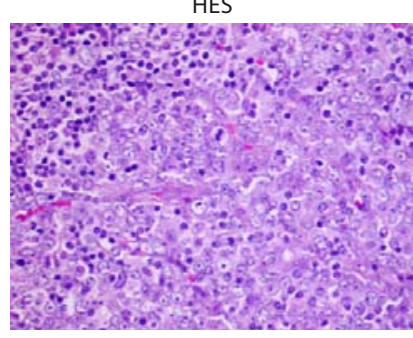

Ki67+

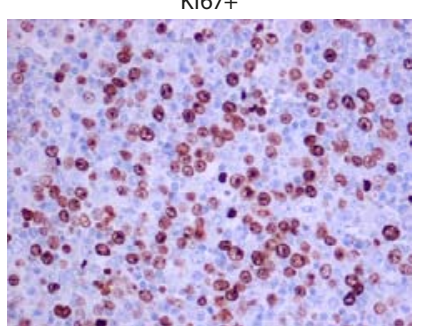

$\mathrm{CD} 45+$

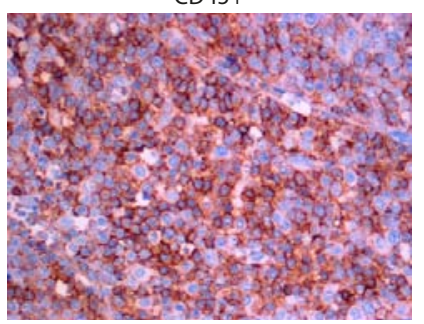

Figure 1. Histological evaluation of lymph node of the patient with myeloid sarcoma in the course of chronic myelogenous leukemia - haematoxylin and eosin staining $(\mathrm{HE})$, immunohistochemicalstaining

The decision to continue the palliative treatment was made. Initially, parameters of the white blood cell system improved. Peripheral lymph nodes became smaller and no longer painful. The patient was considered eligible for radiation treatment, but because of the gradual deterioration in the patient's general condition, the therapy was not performed. The patient's haemoglobin levels decreased, so that she 
required packed red blood cell transfusions. Leukocyte count increased in spite of the raised dosage of hydroxycarbamide and 6-mercaptopurine. In March 2015, the patient died of infectious complications.

\section{DISCUSSION}

Myeloid sarcoma is mostly associated with the recurrence of AML, but it can also occur in patients who have never been diagnosed with myeloproliferative disorder. The literature reports that $87 \%$ of patients diagnosed with isolated myeloid sarcoma subsequently develop bone marrow involvement in a mean time period of 10.5 months. Other patients, however, may never experience progression of the disease [7].

MS is extremely difficult to diagnose. If the patient is diagnosed with AML or another heamatological disease, the diagnosis of MS is relatively easy, but in the case of a primary tumour, the diagnosis poses a great challenge to the pathologist. According to Meis et al. [8], MS was misdiagnosed as a lymphoproliferative disorder in $75 \%$ of cases. Other researchers report that the tumour is undiagnosed in 25$47 \%$ of patients. It is often mistaken for a non-Hodgkin lymphoma (NHL), histiocytic lymphoma, extramedullary haematopoiesis, Ewing's sarcoma, melanoma, anaplastic carcinoma, small-cell lung cancer, inflammation, or other neoplastic proliferation [9].

Diagnostic difficulties often result from the fact that $50 \%$ of myeloid sarcomas are asymptomatic and can occur in any body part $[5,10]$. Symptoms of the disease depend mainly on the location of the tumors. The following signs have been reported in the population of patients with MS: ascites, gastrointestinal bleeding, superior vena cava syndrome, exophthalmos, neurological signs and urinary retention. The most common locations of the tumor include skin, lymph nodes, subcutaneous tissue, bones, retroperitoneal space, mammary gland and eye socket. In women, the ovaries are often affected by the disease. Involvement of the vulva, uterus and vagina have also been reported. It is estimated that the gastrointestinal tract is involved in $6.5 \%$ of cases, producing symptoms such as constipation, haemorrhage, perforation or pain. When the disease affects the respiratory tract it may manifest as interstitial pneumonia, pulmonary nodules, infiltration of bronchial walls or a mediastinal mass. The central nervous system may be affected in the form of extracranial or intracranial tumours $[5,11,12]$. In 2005, Kara et al. [13] described myeloid sarcoma in a very rare location - in the heart. This was a case of relapse of AML after allogeneic stem cell transplantation.

Depending on the level of cellular maturity, the following variants of MS have been defined: blastic (with predominance of myeloblasts), immature (with a mix of myeloblasts and promyelocytes), and differentiated (with promyelocytes and more mature granulocytes). The following variants of MS can be distinguished on the basis of predominant cells: granulocytic, monoblastic, myelomonoblastic, megakaryocytic and erythroblastic [12].

Molecular testing also plays an important role in the diagnostic process. The most commonly described abnormalities associated with myeloid sarcoma include: a $\mathrm{t}(8 ; 21)(\mathrm{q} 22 ; \mathrm{q} 22)$ translocation, trisomy of chromosome 8 and inversion of chromosome 16 (inv16 (p13:q22)). Less common abnormalities are $\mathrm{t}(10: 11), \mathrm{t}(12: 13), \mathrm{t}(12: 22), \mathrm{t}(3: 4)$ and trisomy of chromosome 21. Karyotypic changes are most often found in patients with myeloid sarcoma with bone marrow involvement $[11,14]$.

Isolated myeloid sarcoma is a rarely diagnosed condition. For this reason, there are only a few large randomized trials reliable enough to indicate the best therapeutic option. The treatment depends mainly on the location of the tumour, general condition and age of the patient. Patients diagnosed with isolated myeloid sarcoma and treated with systemic chemotherapy are less likely to develop acute myelogenous leukemia, and the time to transformation to AML is significantly longer. It has also been proved that monotherapy with radiotherapy is not the best therapeutic option. Transformation of MS to AML has often been observed in this group of patients. Long-term results of surgical treatment are poor - the majority of patients experience recurrence of the disease and transformation to AML [14].

Myeloid sarcoma correlated with the blast crisis phase in 9 out of 12 patients diagnosed with chronic myelogenousleukaemia (CML). For this reason, the diagnosis of myeloid sarcoma is an adverse prognostic factor in patients with and without concurrent myeloproliferative disorder [7].

The patient presented in this case report had a long history of CML, and she also had progressive lymphadenopathy before the diagnosis of blast crisis. At first, the enlarged lymph nodes were not painful and but were considered a sign of the disease, but after administration of remission-inducing chemotherapy (2CdA+LD Ara-C), their size increased and they became painful. These symptoms suggested that there was a secondary neoplasm. Interestingly, the re-use of hydroxycarbamideimproved parameters of the white blood cell system reduced the size of the peripheral lymph nodes, and alleviated pain. The patient was considered eligible for radiation treatment, but because of the serious medical condition, the therapy was not performed.

There is no literature data on the effectiveness of the abovementioned treatment regimen, but radiotherapy or surgery used in combination with systemic chemotherapy may be a therapeutic option for MS [14, 15].

A better understanding of the biology of the disease may contribute to the improvement of prognosis and therapeutic methods. Therefore, further research and more case reports of patients with this rare disease are needed.

\section{REFERENCES}

1. Burns A. Observation of surgical anatomy, in Head and Neck. London, England, Royce; 1811.

2. King A. Case of chloroma. Monthly J Med. 1853; 17: 17.

3. Dock G, Warthin AS. A new case of chloroma with leukemia. Trans Assoc Am Phys. 1904; 19(64): 115.

4. Rappaprt H. Tumors of the hematopoietic system, in Atlas of tumor Pathology, Section III, Fasciale 8, Armed Forces Institute Pathology, Washington DC; 1967.

5. Wąsik-Szczepanek E. Mięsak mieloidalny (MM) - występowanie, charakterystyka kliniczna. Acta Haematol Pol. 2008; 39(2): 191-196 (in Polish).

6. Seifert RP, Bulkeley W, Zhang L, Menes M, Bui MM. A practical approach to diagnose soft tissue myeloid sarcoma preceding or coinciding with acute myeloid leukemia. Ann DiagnPathol. 2014; 18(4): 253-260.

7. Jordan RC, Glenn L, Treseler PA, Regezi JA. Granulocytic sarcoma: case report with an unusual presentation and review of the literature. J Oral Maxillofac Surg. 2002; 60(10): 1206-1211.

8. Meis JM, Butler JJ, Osborne BM, Manning JT. Granulocytic sarcoma in nonleukemic patients. Cancer 1998; 58(12): 2697-2709. 
9. Klco JM, Welch JS, Nguyen TT, Hurley MY, Kreisel FH, Hassan A, Lind AC, Frater JL. State of the art in myeloid sarcoma. Int J Lab Hematol. 2011; 33(6): 555-565

10. Sisack MJ, Dunsmore K, Sidhu-Malik N. Granulocytic sarcoma in the absence of myeloid leukemia. J Am Acad Dermatol. 1997; 37(2 Pt 2): 308-311.

11. Campidelli C, Agostinelli C, Stitson R, Pileri SA. Myeloid sarcoma: extramedullary manifestation of myeloid disorders. Am J Clin Pathol. 2009; 132(3): 426-437.

12. Rup E, Wielowieyska-Szybińska D, Sułowicz D, Sacha T, Wojas-Pelc A, Skotnicki A. Myeloidsarcoma - opis przypadku. Prz Dermatol. 2009; 96: 215-219 (in Polish).
13. Kara IO, Sahin B, Paydas S, Kara B. Granulocytic sarcoma of the heart: extramedullary relapse of acute myeloblastic leukemia after allogeneic stem cell transplantation successfully treated by chemotherapy alone. Leuk Lymphoma. 2005; 46(7): 1081-1084.

14. Yilmaz AF, Saydam G, Sahin F, Baran Y. Granulocytic sarcoma: a systematic review. Am J Blood Res. 2013; 3(4): 265-720.

15. Raucci A, Fontanarosa A, Somma P, Bergaminelli C, Valente T, Rea G. Myeloid sarcoma of rib: An atypical isolated chest finding. Egyptian J Radiol Nucl Med. 2015; 46: 105-109. 